А. М. Самойленко, В. В. Слюсарчук (Ип-т математики НАН Украины, Киев)

\title{
ДИСКРЕТНЫЕ ДИНАМИЧЕСКИЕ СИСТЕМЫ С ИНВАРИАНТНЫМ АСИМПТОТИЧЕСКИ УСТОЙЧИВЫМ ТОРОИДАЛЬНЫМ МНОГООБРАЗИЕМ
}

We obtain conditions of the asymptotic stability for quasiperiodic trajectories of discrete dynamical systems in the case of infinite-dimensional Banach space.

Отримано умови асимптотичної стійкості квазіперіодичних траєкторій дискретних динамічних систем у випадку нескінченговимірного банахового простору.

Рассмотрим динамическую систему, заданнуго разностным уравнением

$$
x(n+1)=X(x(n)), \quad n \geq 0,
$$

где $X: E \rightarrow E-C^{r}$-отображение ( $E-$ произвольное бесконечномерное банахово пространство, $r \geq 2$ ). Предположим, что эта система имеет инвариантное многообразие $M=\left\{x \in E: x=f(\varphi), \varphi \in \mathcal{T}_{m}\right\}$ класса $C^{r}$ ( $\mathcal{I}_{m}-$ m-мерный тор), которое заполнено квазипериодическими траекториями

$$
x(n, f(\varphi))=f(n \omega+\varphi), \quad n \geq 0, \quad \varphi \in \mathcal{T}_{m}
$$

$\left(\omega=\left(\omega_{1}, \ldots, \omega_{m}\right)\right.$ - частотный базис квазипериодической функции $\left.f(t \omega)\right)$. В данной статье выясним, когда инвариантное многообразие $M$ системы (1) будет асимптотически устойчивым. Для решения этой задачи используем некоторые результаты из работы [1].

1. Теорема о представлении системы (1) вблизи многообразия $M$. Потребуем, чтобы многообразие $M$ было диффеоморфным тору $\mathcal{I}_{m}$. Тогда это многообразие, как и тор $\mathcal{T}_{m}$, будет m-мерным многообразием. Пусть $d f_{\varphi}-$ производная отображения $f: \mathcal{T}_{m} \rightarrow E$ в точке $\varphi$, а $T\left(\mathcal{T}_{m}\right)_{\varphi}$ и $T M_{f(\varphi)}$ касательные пространства к многообразиям $\mathcal{T}_{m}$ и $M$ соответственно в точках $\varphi$ и $f(\varphi)$ [2-4]. В силу гомеоморфности $\mathcal{T}_{m}$ и $M$ пространства $T\left(\mathcal{T}_{m}\right)_{\varphi}$ и $T M_{f(\varphi)}$ имеют одинаковую размерность $m$, а отображение $d f_{\varphi}: T\left(\mathcal{T}_{m}\right)_{\varphi} \rightarrow T M_{f(\varphi)}$ является линейным изоморфизмом для всех $\varphi \in \mathcal{I}_{m}$. Конечномерность пространства $T M_{f(\varphi)}$ позволяет представить банахово пространство $E$ (для каждого $\varphi \in \mathcal{I}_{m}$ )в виде прямой суммы $T M_{f(\varphi)}$ и некоторого подпространства $E_{\varphi}[5]:$

$$
E=T M_{f(\varphi)}+E_{\varphi}
$$

Выбор подпространства $E_{\varphi}$ осуществляется таким образом, чтобы проектор $P(\varphi)$ на $E_{\varphi}$ параллельно $T M_{f(\varphi)}[6]$ был $C^{r-1}$-отображением (это возможно в силу конечномерности, компактности и принадлежности классу $C^{r}$ многообразия $M$ ). Соответствующие расщепления пространства $E$ на пространства $T M_{f(\varphi)}$ и $E_{\varphi}\left(\varphi \in \mathcal{T}_{m}\right)$ порождают $C^{r-1}$-отображение $F$, которое каждой паре $(\varphi, h)$ элементов $\varphi \in \mathcal{T}_{m}$ и $h \in E_{\varphi}$ ставит в соответствие вектор $x \in E$ :

$$
x=f(\varphi)+P(\varphi) h .
$$

Это отображение имеет обратное отображение, а именно: если $V_{\delta}\left(\mathcal{I}_{m}\right)=$ $=\left\{(\varphi, h): \varphi \in \mathcal{T}_{m}, h \in E_{\varphi},\|h\|<\delta\right\}$ и $U_{\delta}(M)=\left\{x \in E: \inf _{y \in M}\|x-y\|<\delta\right\}$, то найдется такое число $\varepsilon>0$, что $F V_{\varepsilon}\left(\mathcal{T}_{m}\right) \supset U_{\mu}(M)$ для некоторого числа $\mu>$ 
$>0$ и отображение $F: V_{\varepsilon}\left(\mathcal{T}_{m}\right) \rightarrow F V_{\varepsilon}\left(\mathcal{T}_{m}\right)$ является $C^{r-1}$-диффеоморфйзмом [1].

Следовательно, вблизи многообразия $M$ можно ввести систему координат относительно переменных $(\varphi, h)$ (по этому поводу см. также [7, с. $324-333]$ ) и упростить исследованше системы (1).

В [1] установлена следующая теорема.

Теорема 1. В достаточно малой окрестности лногообразия $M$ систельа (1) относительно перельенных $(\varphi, h) \in \mathcal{T}_{m} \times E_{\varphi}$ представляется в виде

$$
\begin{gathered}
\varphi(n+1)=\varphi(n)+\omega+\Phi(\varphi(n)) h(n)+\Phi_{1}(\varphi(n), h(n)), \\
h(n+1)=H(\varphi(n)) h(n)+H_{1}(\varphi(n), h(n)), \quad n \geq 0,
\end{gathered}
$$

где $\Phi(\varphi): E_{\varphi} \rightarrow T\left(\mathcal{T}_{m}\right)_{\varphi+\omega}, H(\varphi): E_{\varphi} \rightarrow E_{\varphi+\omega}\left(\varphi \in \mathcal{T}_{m}\right)$ - линейные отображения, определенные равенствали

$$
\begin{gathered}
\Phi(\varphi)=(d f)_{\varphi+\omega}^{-1}(I-P(\varphi+\omega))(d X)_{f(\varphi)} P(\varphi), \\
H(\varphi)=P(\varphi+\omega)(d X)_{f(\varphi)} P(\varphi),
\end{gathered}
$$

$\Phi_{1}(\varphi, h)$ и $H_{1}(\varphi, h)$ - в общель случае нелинейные отображения, удовлетворяюшиие соотношенио

$$
\sup _{(\varphi, h) \in \tau_{m} \times E_{\varphi},\|/ h\|<\varepsilon}\left(\left\|\Phi_{1}(\varphi, h)\right\|+\left\|H_{1}(\varphi, h)\right\|\right)=o(\varepsilon)
$$

при $\varepsilon \rightarrow 0$, и отображения $\Phi, \Phi_{1}, H, H_{1}$ являются $C^{r-1}$-отображенияли.

Это утверждение является исходным для дальнейших исследований. IX] него, в частности, вытекает, что изучение асимптотической устойчивости инвариантного многообразия $M$ системы (1) сводится к изученито асимптотической устойчивости системы (2) по отношениг к части переменных, точнее по отношенико к переменной $h$ (такого типа устойчивость движения рассматривалась B [8]).

2. Необходимье и достаточпые условия асимптотихеской устойчивостия мюогообразия $M$ в случае жулевых $\Phi, \Phi_{1}$ и $H_{1}$. Мы предполагаем, что $\Phi$, $\Phi_{1}$ и $H_{1}$ принимагот нулевые значения на мғожестве $V_{\mu}\left(\mathcal{T}_{m}\right)$ с достаточно малым $\mu>0$. В этом случае система (2) принимает вид

$$
\begin{gathered}
\varphi(n+1)=\varphi(n)+\omega, \\
h(n+1)=H(\varphi(n)) h(n), \quad n \geq 0,
\end{gathered}
$$

если $(\varphi(n), h(n)) \in V_{\mu}\left(\mathcal{I}_{m}\right)$. Поскольку

$$
\varphi(n)=n \omega+\varphi_{0}, \quad n \geq 0,
$$

где $\varphi_{0} \in \mathcal{T}_{m}$, то, очевидно, инвариантное многообразие $M$ системы (1) является аскмптотически устойчивым тогда и только тогда, когда равномерно по $\varphi_{0} \in \mathcal{I}_{m}$ асимптотически устойчкво нулевое решенше разностного уравнения

$$
h(n+1)=H\left(n \omega+\varphi_{0}\right) h(n), \quad n \geq 0,
$$

с квазипериодическим [9] операторным коэффициентом $H\left(n \omega+\varphi_{0}\right)$ (здесь в "равностепенно по $\varphi_{0} \in \mathcal{I}_{m}$ асимптотически устойчиво" вкладывается следующий смысл: найдется такая функция $g(n) \rightarrow 0$ при $n \rightarrow+\infty$ со значениями в $(0,+\infty)$, что для каждого решения $h\left(n, \varphi_{0}, h_{0}\right)$ уравнения (4), для которого $h\left(0, \varphi_{0}, h_{0}\right)=h_{0}$, выполняется соотношение 


$$
\left\|h\left(n, \varphi_{0}, h_{0}\right)\right\| \leq g(n)\left\|h_{0}\right\|
$$

для всех $n \geq 0$ и $\left.\varphi_{0} \in \mathcal{T}_{m}\right)$.

Учитывая последнее замечание, докажем следугщее утверждение.

Теорема 2. Пусть $\Phi(\varphi)=0, \Phi_{1}(\varphi, \dot{h})=0$ u $\mathrm{H}_{1}(\varphi, h)=0$ для всех $(\varphi, h) \in V_{\mu}\left(\mathcal{T}_{m}\right)$. Тогда инвариантное льногообразие $M$ систельь (1) асихптотически устойчиво тогда и только тогда, когда

$$
\lim _{n \rightarrow \infty} \max _{\varphi \in \mathcal{T}_{m}}\|H(n \omega+\varphi) H((n-1) \omega+\varphi) \ldots H(\omega+\varphi) H(\varphi)\|=0 .
$$

Доказательство. Пусть многообразие $M$ асимптотически устойчиво, т. е. найдется число $\varepsilon_{0}$ такое, что каждому $\varepsilon \in\left(0, \varepsilon_{0}\right)$ будет соответствовать такое $\delta \in\left(0, \varepsilon_{0}\right]$, что для решения $\varphi(n)=\varphi\left(n, \varphi_{0}, h_{0}\right), h(n)=h\left(n, \varphi_{0}, h_{0}\right)$ системы (3), где $\left(\varphi_{0}, h_{0}\right) \in V_{\delta}\left(\mathcal{T}_{m}\right)$ и $\varphi(0)=\varphi_{0}, h(0)=h_{0}$, выполняются соотношенкя

$$
\sup _{n \geq 0,\left(\varphi_{0}, h_{0}\right) \in V_{\delta}\left(\tau_{m}\right)}\left\|h\left(n, \varphi_{0}, h_{0}\right)\right\| \leq \varepsilon
$$

II

$$
\lim _{n \rightarrow+\infty} \sup _{\left(\varphi_{0}, h_{0}\right) \in V_{\delta}\left(\mathcal{T}_{m}\right)}\left\|h\left(n, \varphi_{0}, h_{0}\right)\right\|=0 .
$$

Поскольку в достаточно малой окрестности многообразия $M$ система (1) относительно переменных ( $\varphi, h)$ представляется в виде (3) (мы предполагаем, что $\Phi(\varphi)=0, \Phi_{1}(\varphi, h)=0$ и $H_{1}(\varphi, h)=0$ для всех $\left.(\varphi, h) \in V_{\varepsilon_{0}}\left(\mathcal{T}_{m}\right)\right)$, то для каждой точки $\left(\varphi_{0}, h_{0}\right) \in V_{\delta}\left(\mathcal{I}_{m}\right)$ и $n \geq 0$ для $h\left(n, \varphi_{0}, h_{0}\right)$ выполняется равенство

$$
h\left(n, \varphi_{0}, h_{0}\right)=H\left((n-1) \omega+\varphi_{0}\right) \ldots H\left(\omega+\varphi_{0}\right) H\left(\varphi_{0}\right) h_{0} .
$$

Отсюда и из (7) вытекает соотношение (5), если учесть также, что норма $\|A\|$ линейного оператора $A$, действующего в банаховом пространстве $E$, определяется равенством $\|A\|=\sup \{\|A x\|: x \in E,\|x\|=1\}$.

Пусть теперь выполняется соотношение (5). Обозначим через $а$ число

$$
\max _{n \geq 0} \max _{\varphi \in \mathcal{T}_{m}}\|H(n \omega+\varphi) H((n-1) \omega+\varphi) \ldots H(\omega+\varphi) H(\varphi)\| .
$$

Тогда, согласно (8), выполняются соотношения (6) и (7), где $\varepsilon$-произвольное число из интервала $(0, \mu)$ и $\delta \in\left(0, \varepsilon a^{-1}\right]$. Итак, многообразие $M$ асимптотически устойчиво.

Теорема 2 доказана.

Замечание 1. В случае, когда замыкание множества $\{f(p \omega): p \geq 0\}$ совпадает с множеством $M$, соотношение (5) выполняется тогда и только тогда, когда

$$
\lim _{n \rightarrow+\infty} \sup _{p \geq 0}\|H((n+p) \omega) H((n+p-1) \omega) \ldots H((p+1) \omega) H(p \omega)\|=0 .
$$

Действительно, согласно равенству $\overline{\{f(p \omega): p \leq 0\}}=M$, непрерывности $H(\varphi)$ на $\mathcal{I}_{m}$ и квазипериодичности $H(p \omega)$ по $p$ множество

$$
A_{n}=\{H((n+p) \omega) H((n+p-1) \omega) \ldots H((p+1) \omega) H(p \omega): p \geq 0\}
$$

всюду плотно в множестве

$$
B_{n}=\left\{H(n \omega+\varphi) H((n-1) \omega+\varphi) \ldots H(\omega+\varphi) H(\varphi): \varphi \in \mathcal{I}_{m}\right\}
$$

для каждого $n \geq 0$. Поэтому если выполняется соотношение (5), то выполня- 
ется соотношение (9). Если соотношение (5) не выполняется, т. е. для некоторых чисел $a>0$ и последовательностей $\left\{\varphi_{k}: k \geq 0\right\} \subset \mathcal{I}_{m},\left\{n_{k}: k \geq 0\right\} \subset N$

$$
\left\|H\left(n_{k} \omega+\varphi_{k}\right) H\left(\left(n_{k}-1\right) \omega+\varphi_{k}\right) \ldots H\left(\omega+\varphi_{k}\right) H\left(\varphi_{k}\right)\right\|>a
$$

для всех $k \geq 0$, то в силу квазщернодичности $H(p \omega)$ по $p$ найдется такая последовательность $\left\{p_{k}: k \geq 0\right\} \subset N$, что

$$
\begin{gathered}
\| H\left(n_{k} \omega+\varphi_{k}\right) H\left(\left(n_{k}-1\right) \omega+\varphi_{k}\right) \ldots H\left(\omega+\varphi_{k}\right) H\left(\varphi_{k}\right)- \\
-H\left(\left(n_{k}+p_{k}\right) \omega\right) H\left(\left(n_{k}+p_{k}-1\right) \omega\right) \ldots H\left(\left(p_{k}+1\right) \omega\right) H\left(p_{k} \omega\right) \|<\frac{a}{2}
\end{gathered}
$$

для каждого $k \geq 0$. Тогда, согласно (10),

$$
\left\|H\left(\left(n_{k}+p_{k}\right) \omega\right) H\left(\left(n_{k}+p_{k}-1\right) \omega\right) \ldots H\left(\left(p_{k}+1\right) \omega\right) H\left(p_{k} \omega\right)\right\| \geq \frac{a}{2}>0
$$

для всех $k \geq 0$, т. е. не выполняется соотношение (9). Итак, соотношения (5) и (9) одновременно выполняготся или не выполняготся.

Равенство $\overline{\{f(p \omega): p \geq 0\}}=M$ равносильно равенству $\overline{\{p \omega: p \geq 0\}}=\mathcal{T}_{m}$ (в силу гомеоморфизма $\mathcal{I}_{m}$ нг $M$ ) и выполняется тогда к только тогда, когда $k_{1} \omega_{1}+k_{2} \omega_{2}+\ldots+k_{m} \omega_{m} \neq 0(\bmod 2 \pi)$ для всех $k_{i} \in N \cap\{0\}, i=\overline{1, m}$, для которых $k_{1}+k_{2}+\ldots+k_{m} \neq 0$ (в силу теоремы Кронекера [10, с. 106]).

3. Достаточне условия асимптотической устойчивости многообразия $M$ (общий случай).

Теорема 3. Для асилптотической устойчивости инвариантного льногообразия М систель (1) достаточно выполнения соотноиения (5).

Доказательство. Пусть выполняется соотношение (5). Возьмем такое число $k \in N$, чтобы

$$
\max _{\varphi \in \mathcal{T}_{m}}\|H((k-1) \omega+\varphi) H((k-2) \omega+\varphi) \ldots H(\omega+\varphi) H(\varphi)\|<\frac{1}{2} .
$$

Тогда для каждого решения $x(n)$ разностного уравнения (1) будет выполняться соотношение

$$
x(n+k)=X^{k}(x(n)), \quad n \geq 0,
$$

где

$$
X^{k}(y)=\underbrace{X(X(\ldots X}_{k \text { pas }}(y) \ldots)) .
$$

Рассмотрим разностное уравнение

$$
Y(n+1)=X^{k}(y(n)), \quad n \geq 0 .
$$

Из автономности системы (13) и соотношения (12) вытекает, что инвариантное многообразие $M$ системы (1) асимттотически устойчиво тогда и только тогда, когда выполняется аналогичное свойство многообразия $M$ по отношению к системе (13). Поэтому докажем выполненте этого свойства.

Согласно теореме 1, в достаточно малой окрестности многообразия $M$ систему (13) относительно переменных $(\varphi, h) \in \mathcal{I}_{m} \times E_{\varphi}$ можно представить в виде

$$
\begin{gathered}
\varphi(n+1)=\varphi(n)+k \omega+\Phi^{(k)}(\varphi(n)) h(n)+\Phi^{(k)}(\varphi(n), h(n)), \\
h(n+1)=H^{(k)}(\varphi(n)) h(n)+H^{(k)}(\varphi(n), h(n)), \quad n \geq 0,
\end{gathered}
$$


где $\Phi^{(k)}(\varphi): E_{\varphi} \rightarrow T\left(\mathcal{T}_{m}\right)_{\varphi+k \omega}, H^{(k)}(\varphi): E_{\varphi} \rightarrow E_{\varphi+k \omega} T\left(\varphi \in \mathcal{T}_{m}\right)$ - линейные отображения, определенные равенствами

$$
\begin{gathered}
\Phi^{(k)}(\varphi)=(d f)_{\varphi+k \omega}^{-1}(I-P(\varphi+k \omega))\left(d X^{k}\right)_{f(\varphi)} P(\varphi) \\
H^{(k)}(\varphi)=P(\varphi+k \omega)\left(d X^{k}\right)_{f(\varphi)} P(\varphi)
\end{gathered}
$$

$\Phi^{(k)}(\varphi, h)$ и $H^{(k)}(\varphi, h)$ - в общем случае нелинейные отображения, удовлетворяющие соотношениго

$$
\sup _{(\varphi, h) \in \mathcal{T}_{m} \times E_{\varphi},\|h\|<\varepsilon}\left(\left\|\Phi^{(k)}(\varphi, h)\right\|+\left\|H^{(k)}(\varphi, h)\right\|\right)=o(\varepsilon)
$$

при $\varepsilon \rightarrow 0$, и отображения $\Phi^{(k)}, \Phi_{1}^{(k)}, H^{(k)}, H_{1}^{(k)}$ являтотся $C^{r-1}$-отображениями. Согласно цепному правилу $[4,11]$ для нахождения производных и инвариантности многообразия $M$ относительно отображения $X$ выполняготся равенства

$$
\begin{gathered}
P(\varphi+k \omega)\left(d X^{k}\right)_{f(\varphi)} P(\varphi)=P(\varphi+k \omega)(d X)_{f(\varphi+(k-1) \omega) \ldots} \\
\ldots(d X)_{f(\varphi+\omega)}(d X)_{f(\varphi)} P(\varphi)= \\
=P(\varphi+k \omega)(d X)_{f(\varphi+(k-1) \omega)} P(\varphi+(k-1) \omega) \\
P(\varphi+(k-1) \omega)(d X)_{f(\varphi+(k-2) \omega)} P(\varphi+(k-2) \omega) \ldots \\
\ldots P(\varphi+2 \omega)(d X)_{f(\varphi+\omega)} P(\varphi+\omega) P(\varphi+\omega)(d X)_{f(\varphi)} P(\varphi),
\end{gathered}
$$

т. е. на основании (15)

$$
H^{(k)}(\varphi)=H((k-1) \omega+\varphi) H((k-2) \omega+\varphi) \ldots H(\varphi+\omega) H(\varphi) .
$$

Следовательно, учитывая (11), получаем

$$
\max _{\varphi \in \mathcal{T}_{m}}\left\|H^{(k)}(\varphi)\right\|<\frac{1}{2} .
$$

Возьмем число $\varepsilon_{0}$ таким, чтобы

$$
\left\|H_{1}^{(k)}(\varphi, h)\right\| \leq \frac{1}{4}\|h\|
$$

для всех $(\varphi, h) \in \mathcal{T}_{m} \times E_{\varphi}$, для которых $\|h\| \leq \varepsilon_{0}$ (выбор такого числа возможен в силу (16)). Тогда на основаник неравенств (17) и (18), а также второго уравнения системы (14) убеждаемся в справедлнвости оценки

$$
\|h(n+1)\| \leq \frac{3}{4}\|h(n)\|, \quad n \geq 0,
$$

если $\|h(0)\| \leq \varepsilon_{0}$. Из этой оценки вытекает асимптотическая устойчивость инвариантного многообразия $M$ системы (13), а следовательно, и системы (1).

Теорема 3 доказана.

Замечание 2. Выполнение соотношения (5) не необходимо для асимптотической устойчивости инвариантного многообразия $M$ системы (1). Действительно, для системы разностных уравнений вида (2)

$$
\begin{gathered}
\quad \varphi(n+1)=\varphi(n)+\sqrt{2}, \\
h(n+1)=h(n)-\left(\sin ^{2} h(n)\right) h(n), \quad n \geq 0,
\end{gathered}
$$

инвариантное многообразие $\mathcal{T}_{1} \times\{0\}$ асимптотически устойчиво, поскольку 
точка 0 является притягивающей для отображения $f: R \rightarrow R$, определенного равенством $f(x)=\left(1-\sin ^{2} x\right) x$.

Однако соотношение (5) не выполняется, поскольку $H(\varphi)$ - единичное отображенке для каждого $\varphi \in \mathcal{T}_{1}$.

Замечание 3. Более простое достаточное условие

$$
\sup _{\varphi \in \tau_{m}}\left\|P(\varphi+\omega)(d X)_{f(\varphi)} p(\varphi)\right\|<1
$$

асимптотической устойчнвости инвариантного многообразия $M$ системы (1) рассмотрено в [1]. В случае конечномерного банахового пространства $E$ аналогичное условке пркведено в работе [12].

Замечание 4. Соотношение (5) выполняется, очевидно, тогда и только тогда, когда найдется число $k \in N$, для которого

$$
\max _{\varphi \in \mathcal{T}_{m}}\|H((k-1) \omega+\varphi) H((k-2) \omega+\varphi) \ldots H(\varphi)\|<1 .
$$

Поэтому теорема 3 охватывает случай систем, соответствующих соотношенито (19).

4. Дополнение к статье [1]. В [1] результаты пп. 4, 5 фактически получены с использованием асимптотической устойчивости многообразия $M$ системы (1), которая обеспечнвалась выполнением соотношения (19). Замена этого соотношения соотношенхем (5) улучшает соответствующие результаты. Аналогичные изменения улучшагот и результаты п. 6.

1. Сальойленко А. М., Слюсарчук В. Е., Слюсарчук В. В. Исследование пелинейного разностиого уравнения в банаховом прострапстве в окрестности квазипериодического решения // Укр. мат. журіл. - 1997. - 49, №12. - С. $1661-1676$.

2. Хири М. Дифферепциалыгая топология. -М.: Мир, 1979. -280 с.

3. Борисович Ю.Г., Звягии В.Г., Шермаи П. Б. Топологические методы в теории нелинейных фредтольмовых операторог. - Воропеж: Изд-во Воропеж. уп-та, 1978. - 79 с.

4. Милнор Дж., Уоллес А. Дифферепциалыпая топология. Началынй курс. - М.: Мир, 1972. -279 c.

5. Крейл С. Г. Линейпые уравнения в банаховом пространстве. - М.: Наука, 1971. - 104 с.

6. Като T. Теория возмущений липейпых отераторов. -М.: Мир, 1972. -740 с.

7. Хенри Д. Геометрическая теория полулипейпьх параболических уравпений. -М.: Мир, 1985. -376 c.

8. Рульянцев B. B., Озирапес A. C. Устойчивость и стабилизация движепия по отношениго к части переменных. - М.: Наука, 1987. - 254 с.

9. Сальойлеко $A . M$. Элемегты математической теории мпогочастотных колебапий. - М.: Наука, 1987. $-303 \mathrm{c}$.

10. Левитап Б. М. Почти-периодические фупкции. -М.: Гостехиздат, 1953. - 396 с.

11. Зорич В. А. Математический анализ: В 2-х т. - М.: Наука, 1984. - Т. 2. - 640 с.

12. Сальойенко А. М. Исследовапие дискретпой системы в окрестности квазипериодической траектории // Укр. мат. журіг. - 1992. - 44, №12. - С. 1702 - 1711. 